\title{
Adsorption Materials and Processes for Carbon Capture from Gas- Fired Power Plants: AMPGas
}

\author{
J. A. Arran Gibson, ${ }^{\dagger}$ Enzo Mangano, ${ }^{\dagger}$ Elenica Shiko, ${ }^{\dagger}$ Alex G. Greenaway, ${ }^{\ddagger}$ Andrei V. Gromov, ${ }^{\S}$ \\ Magdalena M. Lozinska, ${ }^{\ddagger}$ Daniel Friedrich, ${ }^{\dagger}$ Eleanor E. B. Campbell, ${ }^{\S, \|}$ Paul A. Wright, \\ and Stefano Brandani* ${ }^{\dagger}$ \\ ${ }^{\dagger}$ School of Engineering, The University of Edinburgh, Edinburgh EH9 3FB, United Kingdom \\ ${ }^{\ddagger}$ EaStCHEM, School of Chemistry, University of St. Andrews, St. Andrews, Fife KY16 9ST, United Kingdom \\ ${ }^{\S}$ EaStCHEM, School of Chemistry, The University of Edinburgh, Edinburgh EH9 3FJ, United Kingdom \\ "Division of Quantum Phases and Devices, School of Physics, Konkuk University, 143-701 Seoul, South Korea
}

Supporting Information

\begin{abstract}
The key challenge in postcombustion capture from gas-fired power plants is related to the low $\mathrm{CO}_{2}$ concentration in the flue gas $(4-8 \%$ by volume). This means that conventional amine processes will result in a relatively high energy penalty, whereas novel adsorbents and adsorption processes have the potential to improve the efficiency of separation. High-selectivity adsorbents are required to achieve relatively high $\mathrm{CO}_{2}$ uptake at low partial pressures, which means that the separation process should be based on either very strong physisorption or chemisorption with thermal regeneration. From the process

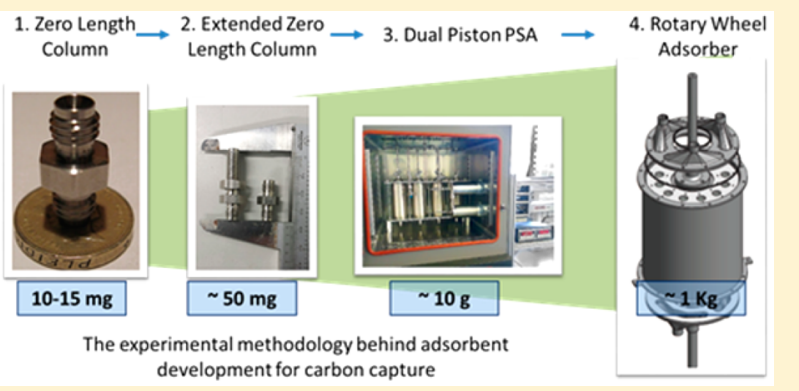
point of view, the main challenge is to develop efficient separation processes with rapid thermal cycles. In this report we present a detailed overview of the methodology behind the development of novel materials and processes as part of the "Adsorption Materials and Processes for Gas-fired power plants" (AMPGas) project. Examples from a wide variety of materials tested are presented, and the design of an innovative bench-scale 12-column rotary wheel adsorber system is discussed. The strategy to design, characterize, and test novel materials (zeolites, amine-containing MOFs, amine-based silicas, amine-based activated carbons, and carbon nanotubes), specifically designed for $\mathrm{CO}_{2}$ capture from dilute streams is presented.
\end{abstract}

\section{INTRODUCTION}

The development and full characterization of novel adsorbents is of primary importance for the effective and economical largescale implementation of carbon capture and storage. There are several challenges associated with the separation of $\mathrm{CO}_{2}$ from flue gas. Typically, the concentration of $\mathrm{CO}_{2}$ in the flue gas is low, ranging from $4 \%$ for a gas-fired power station to up to $14 \%$ for a coal- fired power plant. ${ }^{1}$ This work concerns $\mathrm{CO}_{2}$ capture from gas-fired power plants. The low concentration of $\mathrm{CO}_{2}$ results in the requirement for an adsorbent that is highly selective at low partial pressures of $\mathrm{CO}_{2}$. An adsorbent must have good cyclic stability over multiple adsorption/desorption cycles, relatively fast kinetics, and a working capacity that is greater than $1 \mathrm{~mol} \mathrm{~kg}^{-1}$.

In a gas-fired power plant with turbines operating under standard conditions, the $\mathrm{CO}_{2}$ concentration in the flue gas is approximately $4 \%$. However, if a percentage of the flue gas is recirculated back through the turbines, then the $\mathrm{CO}_{2}$ partial pressure at the outlet can be increased to approximately $8 \%$ without detrimentally affecting the efficiency of the turbines. ${ }^{1,3,4}$ Because the $\mathrm{CO}_{2}$ concentration is higher, the adsorbent will adsorb a greater quantity of $\mathrm{CO}_{2}$; therefore, the required size of the separation unit, the energy penalty, and hence the cost of the carbon capture process can be reduced. ${ }^{3}$

Over the years, several different types of materials have been proposed and investigated for the separation of $\mathrm{CO}_{2}$ from dilute gas streams. These include zeolites, metal-organic frameworks, and amine-modified activated carbons (ACs) and silicas. There are several recent comprehensive reviews that compare the attributes of different solid adsorbents for carbon capture. ${ }^{2,5-7}$ It is clear that an adsorbent is required to have a high affinity and selectivity for $\mathrm{CO}_{2}$ at low partial pressures to be economically viable.

To determine fully a material's potential in a capture process, a number of different parameters have to be established using a variety of different techniques. It is important to be able to test a small amount of laboratory-synthesized adsorbent (50-100 $\mathrm{mg}$ ) and rapidly establish whether the material merits further study. The initial step in the full characterization of a potential

Received: January 4, 2016

Revised: February 27, 2016

Accepted: March 16, 2016

Published: March 16, 2016 


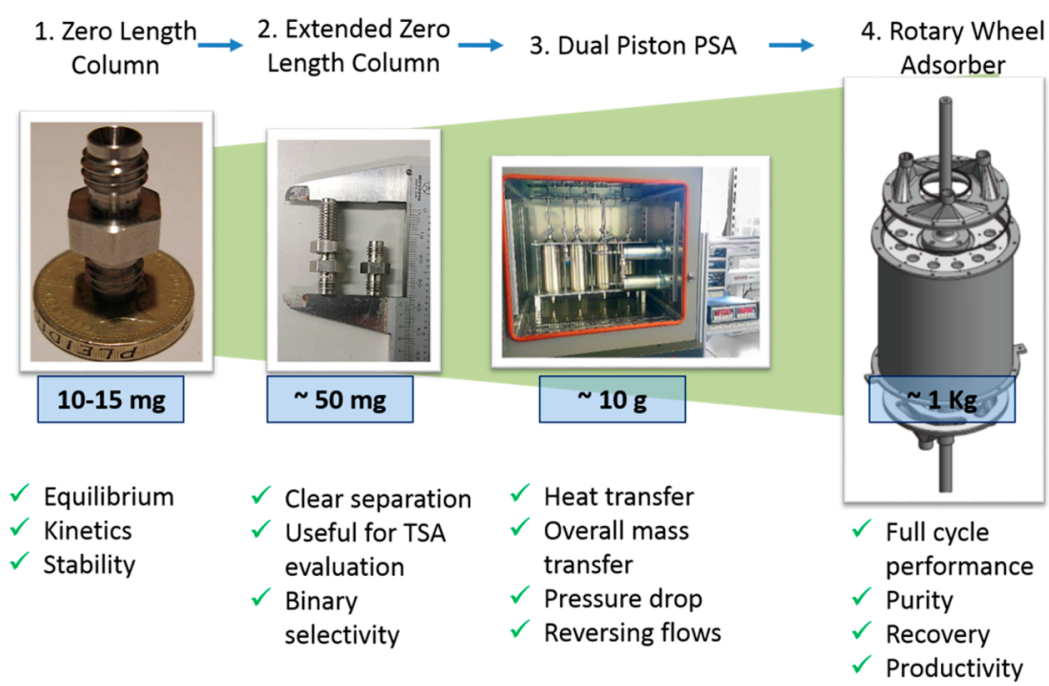

Figure 1. Experimental techniques used to characterize materials at different stages of up-scaling. Adsorbent properties investigated by each technique are detailed below.

material requires the rapid ranking of the $\mathrm{CO}_{2}$ uptake at conditions relevant to the carbon capture process. If a material is deemed to have promising initial properties, i.e., high affinity for $\mathrm{CO}_{2}$, then further tests are carried out to establish the relevant kinetic, equilibrium, and stability characteristics of the material. The material then needs to be scaled up to test its separation performance directly. If all the required performance parameters are determined, then an accurate model can be implemented to evaluate fully the costs and performance of a large-scale separation process, and the true potential of the novel material can be predicted. The general methodology used to characterize the materials at different stages of up-scaling are illustrated in Figure 1.

There have been several different adsorption separation processes proposed for carbon capture. A recent review by Abanades et l. $^{2}$ discusses in detail the applicability of the various process configurations to specific separation conditions. A few examples of separation technologies include pressureswing adsorption (PSA), vacuum pressure-swing adsorption (VPSA), temperature-swing adsorption (TSA), and electricswing adsorption (ESA).

In a PSA process, the adsorbent selectively adsorbs the component that strongly interacts with the surface while allowing the majority of the weakly adsorbed components to pass through the adsorption bed. The adsorbent is then regenerated by removing the strongly adsorbed component at low pressure. To achieve a high-purity product stream, multiple stages may be required. PSA systems have been widely documented and are used in a variety of separation processes on an industrial-scale. ${ }^{8}$ VPSA works on a similar basis to that of PSA except the adsorbent is regenerated at a pressure below atmospheric. A postcombustion capture process involving pressure swing would have to be a VPSA process because the cost of compressing all the flue gas to high pressure is too great. $^{2}$ TSA involves selective adsorption of one component at low temperature followed by a second step where there is a rapid temperature rise of the adsorbent, increasing the mole fraction of the adsorbed component in the gas phase, hence purifying the product stream. . $^{9}$ An ESA process can rely on an increase in temperature of the packed bed by joule heating of the adsorbent as a result of applying a potential difference across it, which in turn results in the desorption of the adsorbed component. ${ }^{10}$ The choice of adsorbent is critical in determining the optimum process to implement for a particular scenario and establishing the lifetime cost of the process.

To capture $\mathrm{CO}_{2}$ from dilute gas streams (4-8\%), strong highly selective adsorbents are required. Such adsorbents have a very steep isotherm profile in the low-pressure region (Figure 2a). As a result, to remove the adsorbed $\mathrm{CO}_{2}$ via a PSA process,

(a)

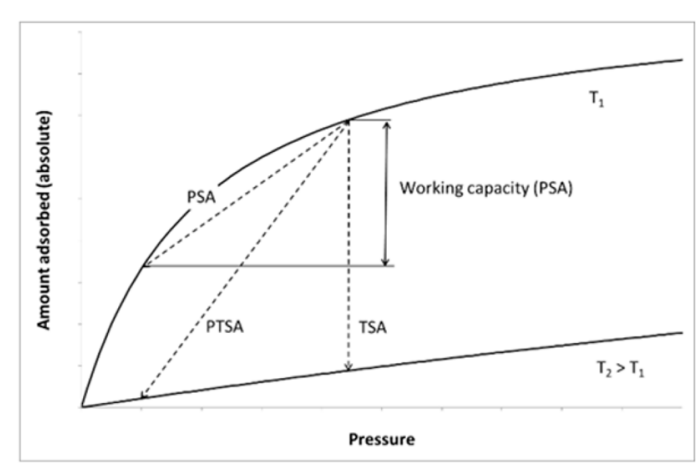

(b)

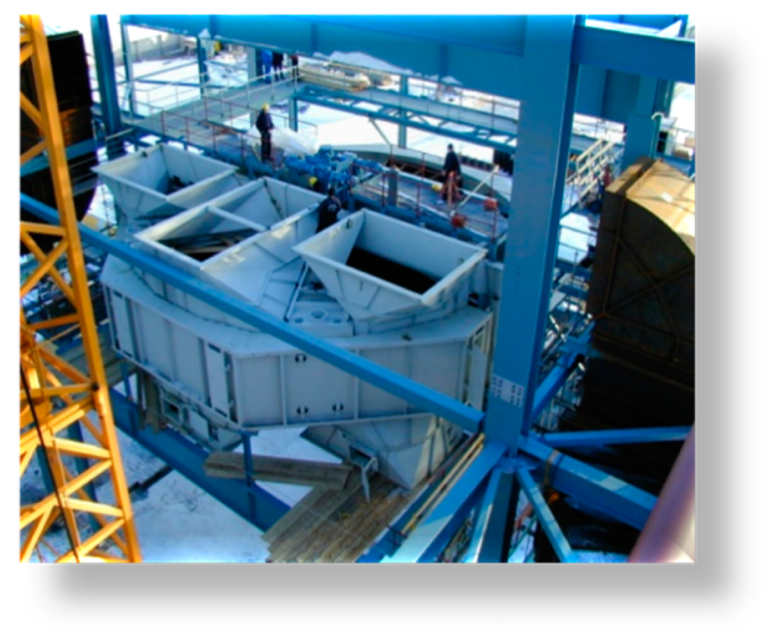

Figure 2. (a) Adsorption isotherms at different temperatures and working capacities. ${ }^{5}$ (b) Industrial rotary heat exchanger, courtesy of Howden. 
very low vacuum pressures are required, which have a significant associated cost. To achieve the target of $90+\%$ recovery and $95+\%$ purity of $\mathrm{CO}_{2}$ from the capture process is not feasible by VPSA in this case. ${ }^{5}$ Through a temperatureswing process, the adsorbent can be regenerated at elevated temperature and can result in adsorbents with significant working capacities. To minimize the size of the adsorption unit, a rapid temperature-swing adsorption (RTSA) process, where the adsorbent can be rapidly cycled through adsorption, regeneration, and cooling steps, is required. A rotary wheel adsorber is well-suited to deliver on these requirements and is therefore considered in this study.

The principle of a rotating adsorption unit has been commercialized by Seibu Giken Co., Ltd., in the form of a honeycomb rotor for the dehumidification of air. ${ }^{11}$ Because a large volume of gas must be treated for cabon capture, an industrial rotary wheel adsorber would be similar in design to a Howden rotary wheel heat exchanger as shown in Figure2b. The adaption of this familiar technology would enable efficient separation of $\mathrm{CO}_{2}$ via RTSA. Inventys has proposed the VeloxoTherm process for carbon capture based on this technology. ${ }^{12}$

The aim of this project was to design materials and processes that could be implemented in a postcombustion carbon capture unit that would separate carbon dioxide from the flue gas from a gas-fired power station. The work was carried out at the interface of chemistry and chemical engineering with the adsorbents' design and properties directly tailored for application in a RTSA process. In this report we present an overview of the methodology used to select suitable adsorbents and show examples from a variety of the tested materials. The development of several novel adsorbents for $\mathrm{CO}_{2} / \mathrm{N}_{2}$ separation and the various experimental measurements that must be conducted to collect the material parameters required to implement a detailed adsorption model are discussed. The validity of the adsorption model can then be verified through the development of a novel lab-scale 12-column rotary wheel adsorber. This in turn validates the model to predict performance at full scale.

\section{ADSORPTION SIMULATOR: CYSIM}

Throughout every stage of the adsorbent characterization, the adsorption properties were simulated by an in-house-developed adsorption simulator, Cysim. ${ }^{13}$ The simulator is very flexible and enables virtually any combination of units (including valves, columns, splitters, and mixing tanks) to be used to simulate an adsorption system. The mass balance and heat balance are accurately modeled, resulting in an accurate prediction of pressure, temperature, and composition profiles of adsorption experiments. Through the Cysim simulator, a complete model can be implemented, taking into account a wide variety of rate limiting mechanisms. The full model hierarchy is visualized in Figure 3.

To simulate fully the adsorption process, many physical parameters are required. These are determined starting from the properties characteristic of a specific material by measuring the equilibrium and kinetic parameters from zero-length column (ZLC) experiments. Having established which controlling mass transfer resistance dominates the separation process, it is possible to select the correct model that reproduces the dynamic response of the column correctly while minimizing the numerical effort from the options available in Cysim (Figure 3). Pressure drop correlations and

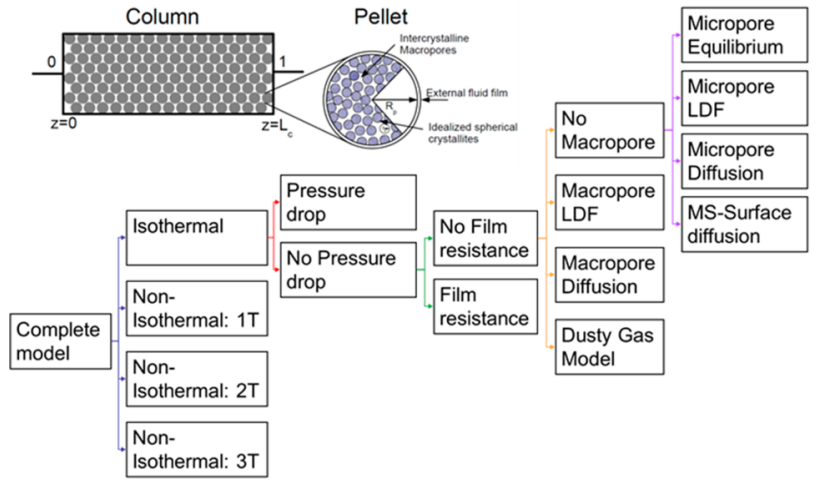

Figure 3. Available adsorption models implemented by Cysim. All the submodels shown for the isothermal case apply to the nonisothermal cases.

heat transfer parameters are validated using the dual-piston PSA experiment. The approach of progressively determining from different experiments the physical parameters that are all correlated using Cysim allows us to establish the predictive ability of the adsorption column model which is at the heart of the simulations of an adsorption separation process.

\section{TESTING NOVEL MATERIALS}

The experimental approach followed in this work has been designed to assess as efficiently as possible the adsorption properties of novel materials. Figure 1 illustrates the experimental approaches that were followed. First, materials are ranked using the ZLC, a technique that requires a few milligrams of sample (typical of laboratory synthesis); then, promising samples are progressively scaled-up to be investigated by a lab-scale rotary wheel process that requires $1-2 \mathrm{~kg}$ of adsorbent. Each of the successive techniques provides further information, which allows the implementation of a detailed model to fully assess and optimize the material's performance in an appropriate process. The background to the various techniques, ZLC, extended ZLC, and dual-piston PSA is detailed below. In addition, the development of a novel labscale rotary wheel adsorber is reported for the first time.

3.1. Ranking of Materials: ZLC. A variety of different techniques exist in the literature to assess the adsorption properties of adsorbents. Techniques include breakthrough experiments, thermogravimetric analysis, volumetric analysis, and the ZLC technique. All these techniques have their advantages; however, the ZLC is a powerful technique for the initial ranking of adsorbents. ${ }^{14,15}$ It was first proposed by Eic and Ruthven ${ }^{16}$ in 1988 as a novel technique to study diffusion in zeolites. However, it has since been used to investigate a wide range of different properties and materials. ${ }^{15} \mathrm{~A}$ welldesigned ZLC setup will quickly rank materials, require small amounts of sample (5-15 mg), provide kinetic information, and enable materials to be tested with impurities such as $\mathrm{SO}_{x}$, $\mathrm{NO}_{x}$, and water. The basic setup has been detailed in several previous publications. ${ }^{14,17}$ In a typical experiment a small mass of sample $(5-15 \mathrm{mg})$ is packed in an $1 / 8$ in. Swagelok union and regenerated at high temperature under inert gas flow. The sample is cooled to the temperature of interest and then equilibrated with a gas stream containing a known partial pressure of sorbate in a carrier gas $\left(\mathrm{He}\right.$ or $\left.\mathrm{N}_{2}\right)$. The gas stream is then switched to a stream of pure carrier gas, and the desorption profile of the adsorbed component is monitored by 
a mass spectrometer. The basic theory has been discussed by $\mathrm{Hu}$ et al. ${ }^{14}$ who used such a system to rapidly rank the $\mathrm{CO}_{2}$ capacity of various metal-organic frameworks (MOFs) and zeolites. In addition, if the system is operated in a regime where it is under equilibrium control, then it is possible to extract the equilibrium isotherm. ${ }^{17-19}$ In this project, the $\mathrm{CO}_{2}$ capacities of the materials were ranked at a concentration of $10 \% \mathrm{CO}_{2}$. Because the ZLC experiment allows the determination of the adsorption isotherm up to the initial concentration, $10 \% \mathrm{CO}_{2}$ by volume was selected as a result of it covering the full concentration range of interest $\left(4-8 \% \mathrm{CO}_{2}\right)$ for carbon capture from gas-fired power plants.

In Figure 4a, sample results of ZLC measurements on several novel materials are shown. The plot shows the ZLC desorption
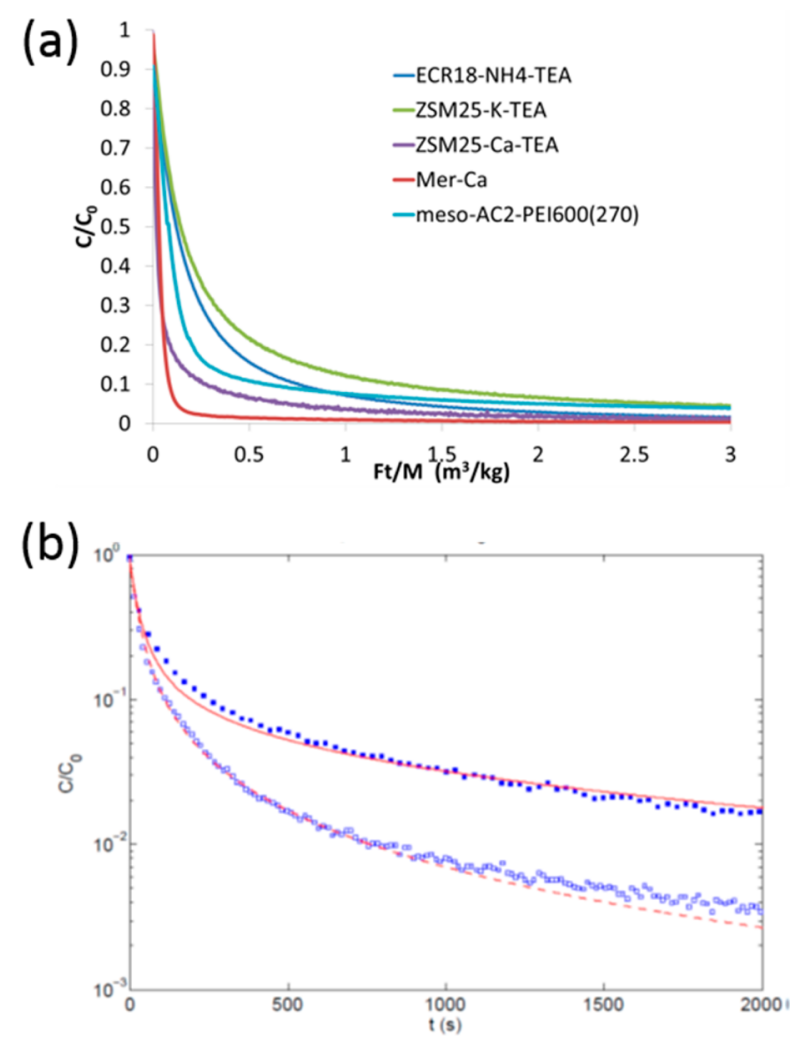

Figure 4. (a) ZLC Ranking of adsorbents at conditions of interest for carbon capture (Zeolites: $35{ }^{\circ} \mathrm{C}$, AC: $75{ }^{\circ} \mathrm{C}, 0.1$ bar $\mathrm{CO}_{2}$ partial pressure). (b) Experimental ZLC desorption curves at $35^{\circ} \mathrm{C}$ for the fully and partially (5.6 min) saturated $\mathrm{Na}$,Cs-Rho sample using $2 \mathrm{~cm}^{3}$ $\mathrm{min}^{-1}$ feed flow of $10 \% \mathrm{CO}_{2}$ in $\mathrm{He}$ and desorbing in a $2 \mathrm{~cm}^{3} \mathrm{~min}^{-1}$ flow of He. The experimental data are given in blue as individual points (solid for fully saturated; open for partially saturated); the curves (red) are calculated using a single value for the diffusivity. ${ }^{21}$

curves of the samples as $C / C_{0}$ (dimensionless concentration) versus the volume of gas eluted (flow rate $X$ time) normalized by the mass of sample, $M$. Representing the experimental data in this way is very useful when comparing the adsorption capacity of different materials because the area under the curve is approximately proportional to the amount adsorbed, i.e., the larger the area under the curve, the greater the material's $\mathrm{CO}_{2}$ capacity, allowing a direct comparison of different samples. ${ }^{14}$ The ranked materials include zeolites produced by the University of St. Andrews ${ }^{20}$ and amine-impregnated carbon (meso-AC-PEI600) produced by the University of Edinburgh. Further discussion regarding the adsorbents can be found in section 4. The zeolites were tested at $35{ }^{\circ} \mathrm{C}$ to maximize the potential working capacity of the adsorbents, whereas the impregnated $\mathrm{AC}$ was tested at $75{ }^{\circ} \mathrm{C}$ to increase the rate of reaction in the chemisorption process. Further details regarding the development of novel materials of interest in this project are given in section 5 .

3.2. Kinetics: ZLC. There have been numerous studies in the literature where the ZLC technique has been used to determine the kinetic parameters of various materials. ${ }^{22,23}$ The technique has also been used to determine which mass transfer mechanism controls the system. For example, it has been used to differentiate between a surface barrier diffusion and intracrystalline diffusion mechanism. ${ }^{24,25}$ In the case of pellets, whether the system is under micro- or macrodiffusion control can be determined by varying the carrier gas, i.e., $\mathrm{N}_{2}$ or $\mathrm{He}$, or by changing the radius of the pellet. ${ }^{22}$ Establishing which mechanism is the controlling mechanism is of great importance when designing and optimizing processes for gas separations. For example, $\mathrm{Hu}$ et al. used the ZLC technique to prove that $\mathrm{CO}_{2}$ adsorption on $13 \mathrm{X}$ pellets occurs under macropore diffusion control. ${ }^{22}$ If an adsorbent is under micropore diffusion control, then the size of the pellets can be optimized to lower the pressure drop along the adsorption column and hence lower the energy penalty of the process. ${ }^{22}$ If a system is under macropore diffusion control, then a compromise needs to be made between the transport kinetics and the pressure drop along the column. The versatility of the ZLC technique allows a wide variety of kinetic parameters to be tested. The ZLC experiment can be performed at various flow rates, with different carrier gases and with variable equilibration times, i.e., the partial loading experiment. The partial loading experiment is an essential tool for the unambiguous determination of the adsorption mechanism. ${ }^{24}$ In this experiment the sample is purposely exposed to the feed mixture for a time significantly lower than the one needed to reach full equilibrium. The shape of the desorption curve will be similar to that of the fully saturated experiment but shifted down by a certain amount depending on the loading time. Figure $4 \mathrm{~b}$ shows, for example, the experimental ZLC data for the full and partial loading experiment on $\mathrm{Na}$,Cs-Rho zeolite; included (in red) are the predicted ZLC curves both for the partial and full loading experiment prediction of the model used to validate the value of the diffusivity. ${ }^{21}$ In addition, Cysim, the adsorption simulator, has been recently adapted to estimate automatically isotherm and kinetic parameters from ZLC experiments. ${ }^{17}$

3.3. ZLC Stability Tests. ZLC can also be used to test the stability of adsorbents with respect to flue gas contaminants such as $\mathrm{SO}_{x}, \mathrm{NO}_{x}$, and water. The sample is exposed to a mixture containing the contaminants for few hours, and after regeneration, the normal test is carried out to determine the $\mathrm{CO}_{2}$ uptake. The experiment is repeated cyclically to determine the variation of the $\mathrm{CO}_{2}$ uptake as a function of the amount of contaminants that have been eluted on the sample. Because of the small amount of material used, results can be obtained rapidly to determine if the sample is stable or if pretreatment of the flue gas is needed before the carbon capture unit. A carbon capture system that uses a physical adsorbent will also require a layered bed to remove water before $\mathrm{CO}_{2}$ is adsorbed. ${ }^{2}$ It is therefore important to test the stability of materials exposed to small amounts of water. In the gas-dosing unit of the ZLC system, which is housed in a temperature-controlled oven, ${ }^{14}$ it is possible to dose water vapor by evaporating controlled amounts of water from a purposely designed capsule. The gas 
mixture containing $10 \% \mathrm{CO}_{2}$ and variable amounts of water ( $500 \mathrm{ppm}$ to $1 \%$ ) is then fed to the ZLC column at low flow rates for several hours, and the amount of water eluted is calculated from the integral in time of the gas flow rate times the water concentration. In Figure 5, two MOFs of the type CPO-27, produced by the University of St. Andrews ${ }^{26}$ were exposed to water (1\%); subsequently, the $\mathrm{CO}_{2}$ capacity of the material was measured

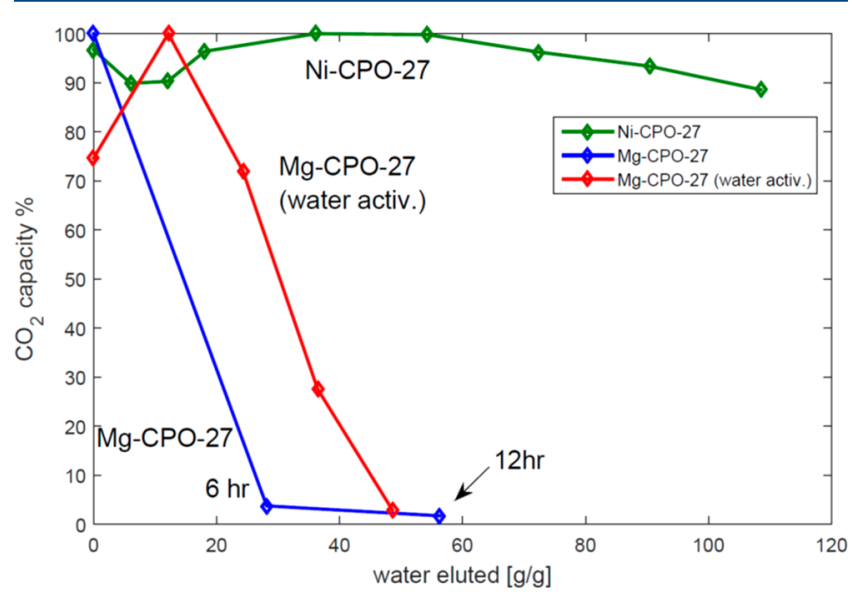

Figure 5. Stability of MOFs on exposure to water tested by the ZLC experiment.

In the presence of water, $\mathrm{Mg}$-CPO-27 tends to deactivate readily, as seen in Figure 5. However, Ni-CPO-17 MOF has a high stability in the presence of water. The higher tolerance of the $\mathrm{Ni}$ center with respect to water is highly desirable to prevent deactivation of the material on exposure to small quantities of water that could be present in the flue gas even after a predrying process. Through the close collaboration between material design and synthesis and adsorbent testing in this project, the potential of the materials as adsorbents can be rapidly fed back to the chemists to aid future material design.

\section{MATERIAL SYNTHESIS AND DEVELOPMENT}

Numerous materials have been reported to have promising characteristics for carbon capture and storage. Throughout the project, there were three classes of material that were of primary interest: zeolites, MOFs, and functionalized carbon materials.

4.1. Zeolites. Zeolites have been widely used as adsorbents because they are very stable and have strong adsorption sites. Zeolites can selectively separate $\mathrm{CO}_{2}$ by a kinetic and/or an equilibrium mechanism. Previously published work has investigated the $\mathrm{CO}_{2}$ adsorption properties of a whole series of univalent cation forms of zeolite Rho $\left(\mathrm{M}_{9.8} \mathrm{Al}_{9.8} \mathrm{Si}_{38.2} \mathrm{O}_{96}, \mathrm{M}=\right.$ $\mathrm{H}, \mathrm{Li}, \mathrm{Na}, \mathrm{K}, \mathrm{NH}_{4}$, and $\left.\mathrm{Cs}\right){ }^{21}$ The $\mathrm{CO}_{2}$ uptake at low partial pressure of $\mathrm{CO}_{2}$ follows the trend of $\mathrm{Li}^{+}>\mathrm{Na}^{+}>\mathrm{K}^{+}>\mathrm{Cs}^{+}$. 21,27 The highest $\mathrm{CO}_{2}$ uptake was obtained on the Li-Rho zeolite $\left(3.4 \mathrm{mmol} \mathrm{g}^{-1}\right.$ at $\left.0.1 \mathrm{bar}, 298 \mathrm{~K}\right)$; however, because of a high affinity to water and the higher price of lithium, the majority of the further investigations were carried out on Na-Rho (3.07 $\mathrm{mmol} \mathrm{g}^{-1}$ at 0.1 bar, $298 \mathrm{~K}$ ), which only had a marginally lower $\mathrm{CO}_{2}$ capacity. These zeolites exhibited interesting adsorption properties with a cation-gating mechanism used to explain the selective transport of $\mathrm{CO}_{2}$ through the windows of the zeolite into the cages. The position of the cation within the cages was observed to be dependent on the pressure of $\mathrm{CO}_{2}$ and the size of the cation. ${ }^{27}$ The postulated gating mechanism ${ }^{28}$ is shown in Figure 6. At low loadings, the diffusivity of the $\mathrm{CO}_{2}$ from the pores was relatively low, potentially limiting their viability in an industrial-scale capture process.

The related synthetic paulingite (Na,H-ECR-18) has also been investigated. Upon dehydration, it was observed that there was a change in symmetry, and the structure distorted to afford closer coordination of the $\mathrm{Na}^{+}$atoms to the $\mathrm{O}$ atoms in the 8membered rings. ${ }^{28}$ The location of the $\mathrm{Na}^{+}$ions again resulted in $\mathrm{CO}_{2}$ adsorption occurring via the "trapdoor" mechanism, which permits separation of the different gas molecules with respect to the strength of the interaction with the cation as opposed to a separation with respect to molecular size. ${ }^{28}$

A recent report by Guo et al. detailed a method to predict the structure of zeolites of increasing complexity and subsequently synthesize them. ${ }^{20}$ Three new zeolite structures ZSM-25, PST20, and PST-25 were structurally resolved and synthesized. These three zeolites have the largest unit cell volume of any zeolites reported to date. $\mathrm{Na}^{+}$-exchanged derivatives of ZSM-25 and PST-20 showed selective uptake of $\mathrm{CO}_{2}$ over both nitrogen and methane. Although the new materials had a lower overall $\mathrm{CO}_{2}$ capacity than that of the previously reported Na-Rho, they exhibited significantly faster kinetics. This was particularly interesting from a process point of view with the large zeolites having a time constant of a few minutes compared to that of the Na-Rho, which had a time constant of several hours. The faster kinetics would result in shorter process cycle times, which could result in a reduction in the required size of the capture plant.

4.2. MOFs. In contrast to zeolites, the structure of a metalorganic framework can be designed, produced, and more easily tailored to a specific application. Both the organic linker and the metal center can be varied to tailor the MOF to have the desired properties. A series of small-pore scandium-based MOFs have exhibited interesting properties for carbon capture. $^{29,30}$ MOF-Sc $_{2} \mathrm{BDC}_{3}$ (BDC $=1$,4-benzenedicarboxylate) was synthesized with a variety of different functional groups on the organic linkers $\left(\mathrm{NO}_{2}\right.$ and $\left.\mathrm{NH}_{2}\right)$. The highest selectivity with respect to $\mathrm{CO}_{2}$ was observed with the aminemodified MOF. ${ }^{31}$ Further investigations into MOF $\mathrm{Sc}_{2}$ (BDC$\left.\mathrm{NH}_{2}\right)_{3}$ utilized a new synchrotron radiation FTIR technique to investigate the adsorption sites and kinetics under dynamic flow. Through the use of polarized light, it was shown that adsorbed $\mathrm{CO}_{2}$ orientates itself along the pore channel. ${ }^{29}$

4.3. Amine-Functionalized Materials. The higher observed $\mathrm{CO}_{2}$ uptake in amine-modified MOFs is of significance because it relates closely to a different approach to adsorbent design. Activated carbons have been used for

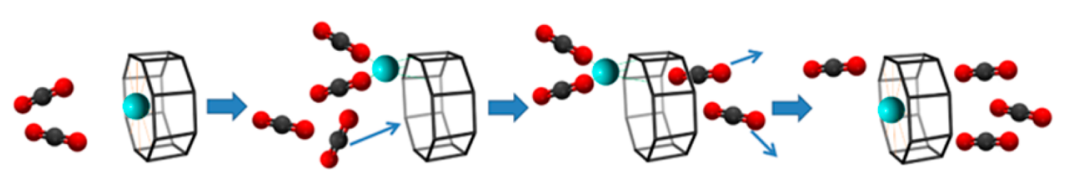

Figure 6. Cation-gating mechanism provides additional selectivity equilibrium and kinetics. 
decades in a variety of separation processes; they have numerous advantages over other types of adsorbents, including high stability and low cost. However, their affinity for $\mathrm{CO}_{2}$ is generally low, and further functionalization of the surface is required to enhance their selectivity toward $\mathrm{CO}_{2}$ at low partial pressures. The addition of amine moieties introduces a basic group to the adsorbent, which can selectively interact with the $\mathrm{CO}_{2}$. There are two approaches to the introduction of amine groups. The functional groups can be grafted to the surface, or alternatively, the AC can be used as a support and liquid amine can be impregnated into the pore volume. The key advantage of amine-functionalized carbons is that they are still selective to $\mathrm{CO}_{2}$ in the presence of water; there is therefore no need to remove the water completely from the flue gas.

The advantages of grafting the amine to the surface include higher thermal and cyclic stability. Several different amines (EDA, TETA, DETA, and PEI) were grafted to a variety of different carbon materials (microporous-AC, mesoporous-AC, and MWCNTS) using a two-step functionalization process (Figure S1). Assuming that two amine groups are required to react with one $\mathrm{CO}_{2}$ molecule, then it was possible to incorporate up to $5 \mathrm{~mol}$ of active sites per kilogram of adsorbent by grafting on amine functionalities. However, after ranking the materials by the ZLC technique, no significant enhancement in the uptake of $\mathrm{CO}_{2}$ was observed for the functionalized materials. When an equivalent number of active sites are incorporated to the $\mathrm{AC}$ via wet impregnation the $\mathrm{CO}_{2}$ capacity was approximately $0.25 \mathrm{~kg} \mathrm{~mol}^{-1}$. ${ }^{32}$ Therefore, although basic groups have been introduced to the surface, without a well-defined microporous structure to supplement the amine- $\mathrm{CO}_{2}$ interaction, it would appear that at present amine-grafted carbons are unable to uptake a comparable amount of $\mathrm{CO}_{2}$ to the reported MOFs and zeolites. This result was in agreement with reports on mesoporous silicas, where the enhancement in the $\mathrm{CO}_{2}$ capacity was in general lower for grafting amines to the surface than that for wet impregnation. ${ }^{6}$

As a result, ACs with varying pore structure (microporous and mesoporous) were impregnated with different polyamines. ${ }^{32}$ A greatly enhanced selective uptake of $\mathrm{CO}_{2}$ was observed for all the impregnated materials (Figure $7 \mathrm{~b}$ ) over that of the raw AC, which had a capacity of $0.15 \mathrm{mmol} \mathrm{g}^{-1}$. It was possible to incorporate a greater number of amine groups into the material by wet impregnation than by grafting the amines to the surface. It was observed that mesoporous AC provided a better support for impregnated amine because of the greater accessibility of the active sites. There was a trade-off between the stability of the polyamine and the efficiency with which it reacted with the $\mathrm{CO}_{2}$. Further carbon materials have been synthesized using the same templating method as previously reported. ${ }^{32}$ Silica with a larger average pore size $(150 \AA)$ was used as a template in order to produce materials with a larger total pore volume to incorporate a greater weight percentage of PEI, which was shown in the earlier work to be the most stable amine for efficient $\mathrm{CO}_{2}$ uptake. The resulting carbon (mesoAC2) had a larger total pore volume of $3.1 \mathrm{~cm}^{3} \mathrm{~g}^{-1}$ and a BET surface area of $1254 \mathrm{~m}^{2} \mathrm{~g}^{-1}$ compared to a pore volume of 1.1 $\mathrm{cm}^{3} \mathrm{~g}^{-1}$ and a BET surface area of $817 \mathrm{~m}^{2} \mathrm{~g}^{-1}$ for the previously reported material. This allowed a PEI weight percentage of $73 \%$ compared to a previously reported maximum of $54 \%$ to be impregnated into the AC. Initial adsorption measurements were carried out on the material (meso-AC2-PEI600(270)) at $35^{\circ} \mathrm{C}$; however, as can be seen in the ZLC experiments in Figure 7a, the uptake of $\mathrm{CO}_{2}$ after an adsorption time of $3 \mathrm{~h}$ was low at (a)
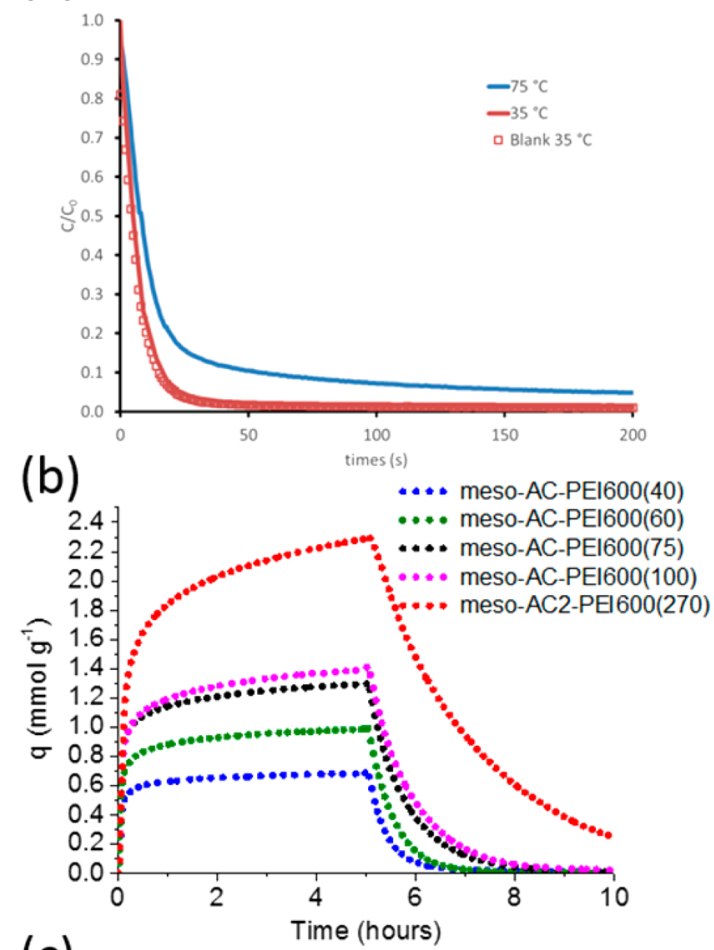

(c)

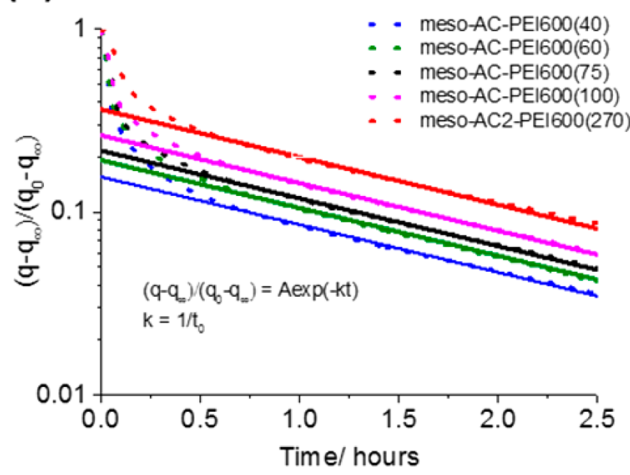

Figure 7. (a) ZLC response of amine-modified activated carbon at 35 and $75{ }^{\circ} \mathrm{C}$ using $5.4 \mathrm{mg}$ of sample; blank system response shown as unfilled squares. Thermal gravimetric analysis of impregnated activated carbons. (b) $\mathrm{CO}_{2}$ adsorption and desorption on activated carbons with various loadings of PEI600. Five hour adsorption step at $75^{\circ} \mathrm{C}, 5 \mathrm{~cm}^{3}$ $\min ^{-1} \mathrm{CO}_{2}, 45 \mathrm{~cm}^{3} \mathrm{~min}^{-1}$, desorption $50 \mathrm{~cm}^{3} \mathrm{~min}^{-1}$. (c) Normalized adsorption step on a semilog plot. Solid line shows long-term asymptote. The number after the sample name corresponds to the weight parts of amine with respect to 100 parts of carbon material.

only $0.17 \mathrm{mmol} \mathrm{g}^{-1}$. The capacity increased significantly to 2.07 mmol $\mathrm{g}^{-1}$ at $75{ }^{\circ} \mathrm{C}$ after $3 \mathrm{~h}$ of adsorption. The higher temperature increases the kinetics of the chemisorption enabling more $\mathrm{CO}_{2}$ to adsorb and desorb within the time frame of the experiment. The higher required operating temperature for the amine-impregnated carbons influences the design of the separation process. It is therefore important that process and material design are carried out in parallel.

Because of the slow kinetics, further measurements were carried out by thermal gravimetric analysis at $75^{\circ} \mathrm{C}$ on a larger sample mass $(\sim 50 \mathrm{mg})$. Over a $5 \mathrm{~h}$ adsorption step, meso-AC2PEI600(270) had a $\mathrm{CO}_{2}$ uptake of $2.3 \mathrm{mmol} \mathrm{g}^{-1}$ (Figure $7 \mathrm{~b}$ ); this was higher than the previously reported impregnated carbons, included in Figure $7 \mathrm{~b}$ for comparison. ${ }^{32}$ Further 
optimization of the pore structure should allow even higher capacities to be reached. In addition, the thermal gravimetric system allows the heat of adsorption to be measured via differential scanning calorimetry. The heat of adsorption for the chemisorption process between the $\mathrm{CO}_{2}$ and the amine was found to be $\sim 90 \mathrm{~kJ} \mathrm{~mol}^{-1}$. ${ }^{32}$

The time constant of the adsorption step was investigated by thermal gravimetric analysis for different loadings of PEI600 in an attempt to understand the reaction kinetics at $75{ }^{\circ} \mathrm{C}$. The experimental data for the adsorption step was normalized using $\frac{q-q_{\infty}}{q_{0}-q_{\infty}}$, i.e., the initial and final value of the adsorbed amounts. The plots of $\frac{q-q_{\infty}}{q_{0}-q_{\infty}}$ versus time on a semilog plot are shown for various loadings of PEI in Figure 7c. It can be seen that in the long-time region the response of each sample approaches an exponential decay. The time constant $\left(t_{0}\right)$ is approximately $5980 \mathrm{~s}$ for all the different loadings of PEI. Because the slope was independent of the amount of amine loaded on the sample, this gradual uptake of $\mathrm{CO}_{2}$ can be attributed to the amine. It was likely that this kinetically limited uptake was a result of the dissolution of the $\mathrm{CO}_{2}$ into the liquid amine in a manner similar to the mechanism proposed by Zhao et al. for impregnated mesoporous silicas. ${ }^{33}$ The transport to the remaining active sites through the liquid amine would appear to be rate-limiting at higher loadings. During desorption, the lower the amine loading, the faster the desorption process occurs. When there is less amine in the pores, a larger percentage of the amine is exposed to the gas environment. This results in a faster release of the adsorbed $\mathrm{CO}_{2}$.

It is clear that the wet-impregnation technique introduces a significantly larger number of basic amine groups to the material compared to grafting techniques, which in turn results in significantly higher $\mathrm{CO}_{2}$ capacities. This is despite the significant loss in pore volume and accessible surface area of the adsorbent when impregnated with polyamine. Solid adsorbents impregnated with amine combine the advantage of the high selectivity of a liquid-amine carbon capture process with a lower cost of regeneration. Unlike a conventional absorption process that uses amines in an aqueous solution, there is no need to heat a large volume of water during the regeneration step because the heat capacity of the solid is lower than that of the solution; therefore, there is the potential for significant energy savings while a high selectivity for $\mathrm{CO}_{2}$ at low partial pressures is maintained. ${ }^{5}$

\section{EXTENDED ZERO-LENGTH COLUMN: BINARY SELECTIVITY}

The extended zero-length (E-ZLC) column is approximately three times the length of the ZLC at $25.9 \mathrm{~mm}$ and is housed in a $1 / 8$ in. Swagelok bulkhead connector (shown in Figure 8), allowing the use of the same setup developed for the traditional ZLC experiment. This allows more sample to be packed in the adsorption column to achieve a clear separation of components in a binary mixture and determine the binary adsorption selectivity. The advantage of the E-ZLC over a larger more traditional breakthrough column $(\sim 5 \mathrm{~g})$ is that a relatively small amount of sample is required $(\sim 50 \mathrm{mg})$ and that the column can still be considered isothermal. To assess whether the system was in fact isothermal, a thermocouple was inserted into the center of the E-ZLC and sealed in place with epoxy resin. Breakthrough experiments on commercial zeolite were carried out to determine the temperature profile within the
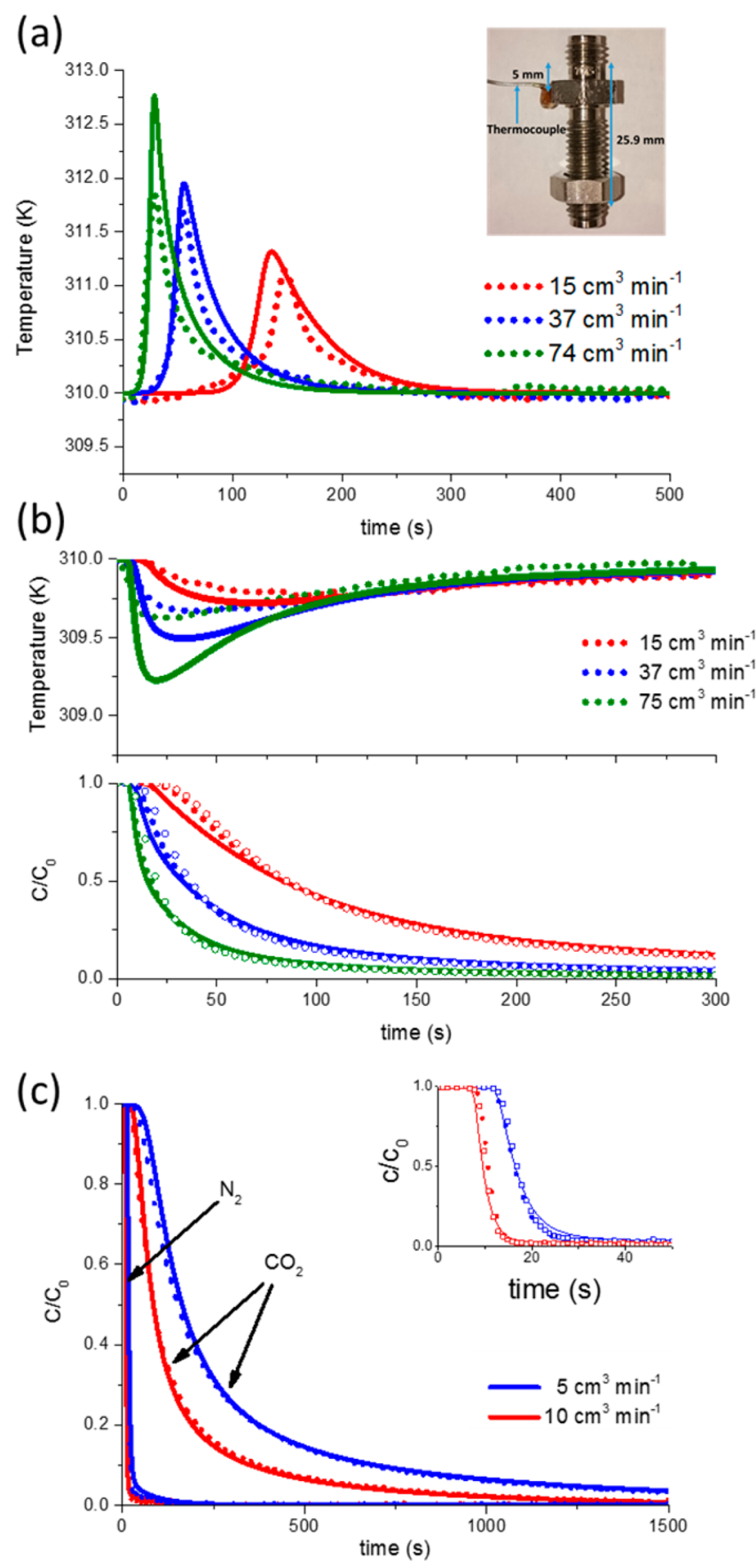

Figure 8. ( $\mathrm{a}$ and b) Breakthrough curves zeolite 13X, $(63.75 \mathrm{mg}, 35$ ${ }^{\circ} \mathrm{C}$; Feed: 0.1 bar $\mathrm{CO}_{2}, 0.9$ bar $\mathrm{N}_{2}$. Purge: $\mathrm{N}_{2}$ ) (a) Experimental temperature profile during adsorption and E-ZLC. Cysim simulation solid lines. (b) Desorption temperature and $\mathrm{CO}_{2}$ profiles with isothermal and nonisothermal Cysim fits. Experimental data: dots, nonisothermal Cysim simulation: solid lines, isothermal Cysim fit: open dots. (c) Binary breakthrough curve $(48.5 \mathrm{mg}$, Feed: 0.1 bar $\mathrm{CO}_{2}, 0.9$ bar $\mathrm{N}_{2}$. Purge $\mathrm{He}$ ) showing clear separation of $\mathrm{N}_{2}$ and $\mathrm{CO}_{2}$. Inset, zoom of $\mathrm{N}_{2}$ breakthrough; blank system response depicted by unfilled squares.

column. To ensure the sample was well-packed around the thermocouple, pellets of $13 \mathrm{X}$ were broken up prior to being packed. The thermocouple was positioned $5 \mathrm{~mm}$ from the end of the column in the gas phase.

From Figure 8a, an experimental spike in the temperature of up to $2.5 \mathrm{~K}$ was observed during the adsorption step. The 
temperature spike was larger at higher inlet flow rate, and the bed returned to an isothermal state within $3 \mathrm{~min}$ as the heat was quickly dissipated to the system. Because of the small diameter of the adsorption column, there was a high rate of heat transfer out of the column; this resulted in a small change in the gas phase temperature.

A much smaller temperature change was observed in the desorption experiments, which is consistent with the fact that under nonlinear conditions desorption is slower than adsorption. Therefore, the desorption experiments are closer to isothermal behavior and require fewer parameters to be modeled. The isotherm parameters were independently collected at three different temperatures on a Quantachrome Autosorb IQ1 volumetric system (Figure S2). A dual-site Langmuir isotherm was used to fit the $\mathrm{CO}_{2}$ isotherms at three different temperatures simultaneously, and the same saturation capacities were used to regress the $\mathrm{N}_{2}$ data to ensure thermodynamic consistency. The resulting isotherm parameters were used in the simulation.

The desorption breakthrough profile was modeled using both an isothermal and a nonisothermal model in Cysim. In the case of the nonisothermal model, the heat transfer coefficient that represents the heat transfer from the fluid phase to the system was adjusted to match the experimentally observed temperature profile. A reasonable fit was achieved between the predicted fluid phase temperature and the experiment (Figure $8 \mathrm{~b})$, considering that a shielded thermocouple was used and the heat transfer dynamics of the thermocouple were not included in the model. Because the temperature change of the fluid during desorption was less than one degree, it was possible to consider the bed to be isothermal. A comparison of the predicted breakthrough profile for the isothermal and nonisothermal case is shown in Figure $8 b$, confirming that the system is sufficiently close to isothermal behavior. There were small simulated differences in the $\mathrm{CO}_{2}$ profile at short times. At $74 \mathrm{~cm}^{3} \mathrm{~min}^{-1}$, where the largest change in temperature was observed, the nonisothermal model provided a more accurate prediction of the experimental breakthrough profile than the isothermal case. However, in the long-time region, there was no noticeable difference between the adsorption models, and both accurately represented the data, confirming the validity of the mass transfer kinetic parameters.

In Figure 8c, the binary desorption curves of $\mathrm{CO}_{2}$ and $\mathrm{N}_{2}$ on $13 \mathrm{X}$ are presented. It can be seen clearly that the E-ZLC enables the separation of a binary mixture by an adsorbent to be investigated. The equilibrium amounts of $\mathrm{CO}_{2}$ and $\mathrm{N}_{2}$ are roughly proportional to the areas under the curves of the desorption experiments. From the inset in Figure $8 \mathrm{~b}$, it can be seen that very little nitrogen was adsorbed on the $13 \mathrm{X}$ because the desorption profile closely matches the blank system response.

$$
S_{\mathrm{ADS}}=\frac{q_{\mathrm{CO}_{2}} / q_{\mathrm{N}_{2}}}{P_{\mathrm{CO}_{2}} / P_{\mathrm{N}_{2}}}
$$

For the system shown in Figure 8, a selectivity for $\mathrm{CO}_{2}$ over $\mathrm{N}_{2}$ of 282 was obtained from the curves simulated in Cysim that match the experimental results.

\section{DUAL-PISTON PSA}

The next stage is to assess the separation potential of an adsorbent in a dual-piston PSA system run at total reflux. ${ }^{33}$ This technique requires approximately $10 \mathrm{~g}$ of sample in pelletized form and tests a single adsorption column in a closed system. The dual-piston PSA system is designed to allow to vary the cycle time from several minutes down to $1 \mathrm{~Hz}$, change the stroke length and the phase angle between pistons, and can be run using pure components and binary mixtures. ${ }^{33}$ This system can be run with a wide range of flow rates, determined by the speed of the pistons (combination of cycle time and stroke length), and generates flows at constant pressure or variable pressure (determined by the offset between the pistons) with reversing direction.

Cysim is used to model the experiment, ${ }^{13}$ and the remaining column parameters are determined in a sequence initially using an inert gas and progressively matching the dynamics of adsorbing gases. This allows us to determine the remaining physical parameters in Cysim, including conditions where Knudsen flow in pellets (vacuum pressures) is the controlling mass transfer mechanism. Heat transfer parameters can be validated using thermocouples placed inside the pellets at two positions along the column axis. The simulations allow us to calculate the concentrations in the two pistons, which in a total reflux configuration are a direct indication of the separation efficiency of the adsorption column.

\section{ROTARY WHEEL ADSORBER: SYSTEM DESIGN}

A rapid cyclic thermal-swing process is required to capture $\mathrm{CO}_{2}$ from a gas-fired power plant by postcombustion capture. This is a result of the low $\mathrm{CO}_{2}$ partial pressure in the flue gas that requires the use of strongly adsorbing, highly selective adsorbents to capture the $\mathrm{CO}_{2}$. However, this means that these materials cannot be regenerated fully by operating the process purely as a VSA system because very low regeneration pressures would be needed. Therefore, thermal swing becomes the more obvious option for a viable adsorption process. The main disadvantage of TSA systems over PSA is the longer cycle time due to the heating and cooling, of the adsorption beds. ${ }^{8,9}$ For this reason, when large volumes of gas are involved, it becomes critical to minimize the cycle time in order to have a competitive separation process. In this regard, a rotating TSA system is a promising technology for the development of rapid TSA cycles. Rotating systems have several advantages compared to the traditional fixed bed approach. Their design, based on the rotary wheel heat exchanger, ensures large heat transfer coefficients in the cold and hot sections. In addition, there is no need of multiple valves, piping and connections keeping the capital costs relatively low. A well-designed rotary adsorber unit can treat large volumes of gases, has efficient heat integration, can perform rapid temperature swings, and will have a low pressure drop through the adsorption beds if structured packings are used. ${ }^{35}$

To investigate the performance of a $\mathrm{CO}_{2}$ separation process based on RTSA, a novel lab-scale rotary wheel adsorber (RWA) has been designed and built. In this system, the heads (inlets and outlets) are fixed, whereas the entire adsorption bed rotates. The RWA system designed in this project is a scaleddown version of the rotary heat exchangers used in industry (Figure2b). The primary purpose of the experimental setup is to produce experimental data to validate the detailed adsorption model and test possible contributions of leakage rates between the sections of the rotary wheel. Using the adsorption parameters previously collected for the adsorbent, the model can be tuned to predict the experimental data generated by the RWA. The model can then be used to predict reliably the performance of a full-scale industrial process. 
The RWA has a stainless-steel structure consisting of 12 columns of $30 \mathrm{~cm}$ length and $2.7 \mathrm{~cm}$ internal diameter, arranged around a rotating shaft as shown in Figure 9. The

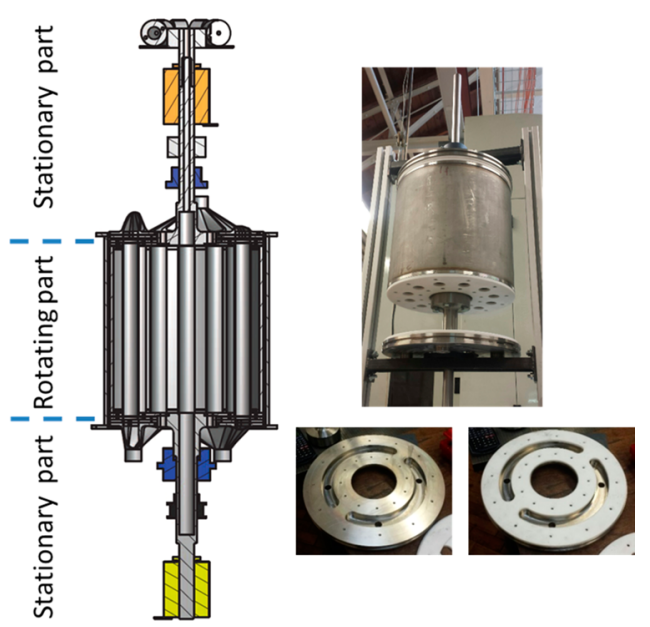

Figure 9. Rotary wheel adsorber.

outer diameter of the rotating structure that contains the columns is $330 \mathrm{~cm}$, and including the supporting structure, the overall apparatus reaches a height of about $1 \mathrm{~m}$. To load the system fully, 12 columns can be packed with between 1 and 2 $\mathrm{kg}$ of adsorbent in total. The choice of this configuration has the clear advantage that each column can be independently removed and tested in a traditional breakthrough experiment and compared with the predictions obtained from Cysim on the basis of the parameters determined in the ZLC, E-ZLC, and dual-piston PSA apparatuses. Two thermocouples have been fitted inside each column to monitor the temperature profile of the bed. The regeneration of the sample is carried out in situ using electrical heating elements inserted inside each column. As the beds are rotating, the power to the heating element is supplied using slip rings that are attached to the bottom of the shaft. In the case of a conductive adsorbent (carbon nanotube based monolith for example), the power supply can be directly connected to the adsorbent, and the adsorbent can be regenerated by resistive heating. During the regeneration, the thermocouples also control the amount of power supplied to the heating elements to avoid the adsorbent overheating. The stationary section on top of the rotating can consists of a stainless-steel sector plate. On top of the plate, there are the inlet connections for the feed and purge gas streams. At the bottom of the plate, a circular groove connects each gas inlet with a fraction of the total number of columns (Figure 9). This defines the sectors of the system, i.e., how many columns are working in adsorption and how many in desorption. Plates with different numbers of columns per sector are available to run the system in different configurations. The bottom section of the setup is identical to the top one. The sealing is ensured using PTFE plates between the sector plates and the rotating can. Perfect sealing between moving parts is extremely difficult to achieve; therefore to minimize possible leaks between the adsorption and desorption sections, the apparatus is designed to work in cocurrent flow, with the gas inlet from the top. A Baldor $\mathrm{ABB}$ motor connected to the top of the shaft allows both the rotation of the system (with a maximum speed of 1 $\mathrm{rpm}$ ) and the accurate determination of the position. The pressure drop across the rotating bed is monitored with a differential pressure transducer connected to the inlet and outlet gas lines. The full control of the unit and the data acquisition is performed using a real time computer (National Instruments CRIO). One of the issues when sending/acquiring data from a moving system is fast and reliable communication with the system. In this case, the system was equipped with a total of 24 thermocouples that need to send the information acquired "on board" to the stationary acquisition system with any time delay kept to a minimum. Given that only a limited number of channels would be available through a physical connection through the shaft, a Light-Fidelity $(\mathrm{LiFi}) / \mathrm{WiFi}$ communication system has been custom designed. The communication device (on top of the shaft) allows acquired data to be sent wirelessly from the thermocouple to a receiver connected to the CRIO using both a traditional WiFi and a novel $\mathrm{LiFi}$ system. $\mathrm{LiFi}$ is a novel communication technology based on visible light. Compared to the available radio frequency spectrum, the visible light spectrum is 10000 times larger, allowing data rates higher than $1 \mathrm{Gps}$, about 100 times faster than conventional $\mathrm{WiFi}{ }^{36,37}$ In addition, visible light does not interfere with radio waves or other electronic equipment, allowing reliable real-time data acquisition. For the specific implementation on the RWA, in order to ensure communication during the rotation of the bed, a LED ring was placed on top of the shaft. This will be one of the first implementations of the LIFi technology on a moving system; therefore, the dual $\mathrm{WiFi} / \mathrm{LiFi}$ device on this system will be used to compare the performance of the two communication systems. Finally, a mass spectrometer is connected online to monitor the outlet gas concentration.

The apparatus is designed to have a wide range of configurations which will be used to validate the Cysim predictions and develop a model for the gas leakage between the different sections in order to arrive at an accurate model needed to predict correctly the purity and recovery of the separation process. This will then allow to evaluate the performance of the full-scale carbon capture process.

\section{ROTARY WHEEL ADSORPTION: PROCESS SIMULATIONS}

In this section, two different benchmark adsorbents were investigated to provide an initial set of ideal separation performance (no gas leakage between sections of the RWA and no water adsorption on the different materials). The two adsorbents of interest were commercial zeolite 13X and MCM41-PEI. These two adsorbents have distinct adsorption properties. $13 \mathrm{X}$ is a physisorbent with relatively high $\mathrm{CO}_{2}$ uptake at low partial pressures, whereas MCM-41-PEI ${ }^{38}$ is a chemisorbent with a high heat of adsorption. Zeolite $13 \mathrm{X}$ is often used as a benchmark material for postcombustion $\mathrm{CO}_{2}$ capture. $^{15}$ Therefore, the comparison of $13 \mathrm{X}$ with a typical chemisorbent becomes a useful case study to assess the viability of the adsorbents in a TSA process.

Figure 10 depicts how the key differences between the adsorbents are related to their equilibrium isotherms and their heats of adsorption. More specifically, isotherms at $308.15 \mathrm{~K}$ and $423.15 \mathrm{~K}$ are shown to evaluate their dependence on the operating temperature, i.e., assuming a process in which adsorption is carried out at low temperature and desorption at high temperature. (At this stage, it was assumed that the chemisorbent is stable at this temperature.) Because the desorption is carried out at high temperature, $\mathrm{CO}_{2}$ (concentrated to nearly pure) can be used as a carrier. This means 


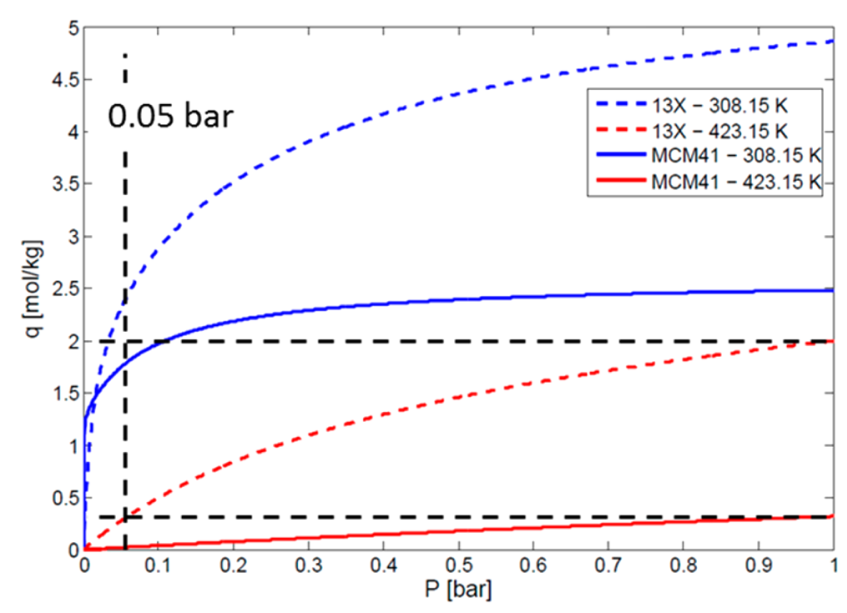

Figure 10. Equilibrium isotherms of $13 \mathrm{X}$ and MCM-41-PEI ${ }^{38}$ at 308.15 and $423.15 \mathrm{~K}$

that although $13 \mathrm{X}$ shows a higher $\mathrm{CO}_{2}$ uptake at low partial pressure $(0.05$ bar $)$ the lower heat of adsorption results in a smaller temperature dependence in the equilibrium isotherm. As a result, the working capacity for $\mathrm{CO}_{2}$ is about $1 / 3$ of the working capacity of the amine-based material, making the latter a promising material for the separation process. Obviously, the evaluation of the equilibrium isotherm is only one of the many parameters that can be used for ranking the potential of adsorbents, and the isotherms should not be the only means upon which the evaluation of the process performance is based. For this purpose, highly detailed simulations are needed.

For this comparison, a simple process configuration was chosen in which the cycle was composed of three steps: adsorption, desorption, and intermediate cooling (Figure 11).

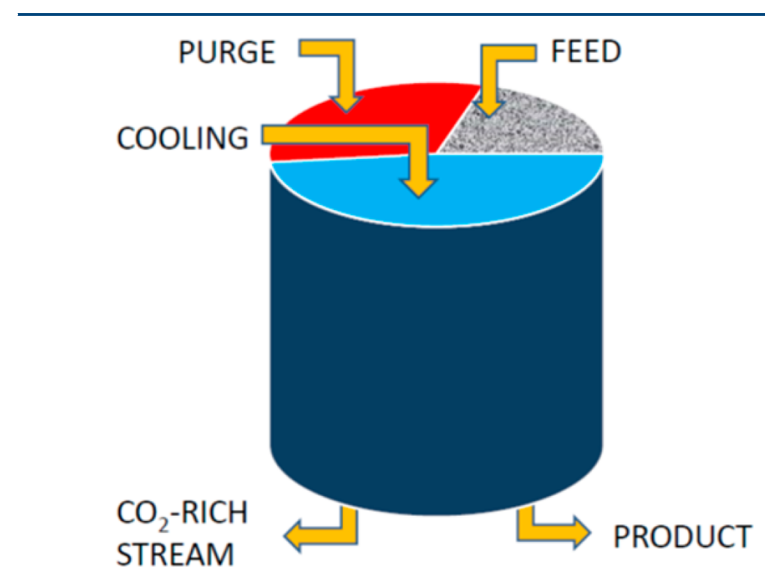

Figure 11. Schematic diagram of the $\mathrm{CO}_{2}$ separation process used in the simulations.

The adsorption inlet feed gas is set at $35{ }^{\circ} \mathrm{C}$ with a concentration of $5 \%$ of $\mathrm{CO}_{2}$ in $\mathrm{N}_{2}$ at atmospheric pressure. The regeneration is carried out using a purge stream of steam at $125^{\circ} \mathrm{C}$. For this step, it was assumed that the steam does not adsorb and no condensation occurs; for this reason, it was imposed that the outlet temperature of the purge is no less than $100{ }^{\circ} \mathrm{C}$ at any time of the process. After the desorption step, the sector is cooled using $\mathrm{N}_{2}$ at $10{ }^{\circ} \mathrm{C}$. For simplicity, it was also assumed that the bed density and void fraction are the same for both adsorbents. Finally, the adsorption kinetics were fast so that the processes were considered to be under equilibrium control. The separation performance depends essentially on the equilibrium isotherm of the two adsorbents.

Figure 12 shows the concentration and temperature profiles for the two cases. It can be seen that as $\mathrm{CO}_{2}$ is adsorbed a temperature wave is generated which travels along the bed. As expected, the intensity of the temperature peak is higher for the amine-supported material because of the higher heat of adsorption. However, from the adsorbed concentration profile it can be seen that the adsorbed amount is significantly lower for this sample. The flow rates and the duration of each step are set so that $90 \%$ recovery of $\mathrm{CO}_{2}$ is achieved in both cases. It was assumed that all the $\mathrm{CO}_{2}$ from the outlet would be recovered by condensing the steam. Table 1 reports some of the significant parameters of the process. The two processes have similar performance in terms of $\mathrm{CO}_{2}$ purity, even though the higher heat of adsorption of the amine-based process would suggest a lower steam $/ \mathrm{CO}_{2}$ capture ratio compared to that of $13 \mathrm{X}$; however, this advantage was counter-balanced by the lower overall uptake of $\mathrm{CO}_{2}$ of the amine-supported material.

As mentioned at the beginning of this section, this is just a simplified study of one of the possible configurations providing an ideal separation benchmark. Clearly, in the case of $13 \mathrm{X}$ the assumption of no water adsorption is not valid, and direct use of steam for the regeneration would not be viable. The aim was primarily to develop the simulation framework in Cysim capable of predicting the separation performance of a RWA system, thus completing the overall methodology of the AMPGas project.

\section{CONCLUSIONS}

Here we have presented a systematic approach to the characterization and development of novel adsorbents for carbon capture from gas-fired power plants. Strong, highly selective adsorbents have been developed that exhibit a high uptake of $\mathrm{CO}_{2}$ at low partial pressure. The methodology behind the characterization and development of the materials and processes has been presented. Various experimental techniques have been discussed, and several examples shown, that enable all the parameters required for a detailed adsorption model to be collected. The detailed adsorption model can then be implemented to predict the separation efficiency and investigate the optimum separation conditions for an adsorbent. The methodology described in this contribution enables an initial rapid screening of many adsorbents from which a subset are scaled up and investigated further. The same adsorption simulator is used at all stages of the approach, allowing us to fix several physical parameters at different stages and to minimize any potential errors of conversion between different data sets. The validity of the model parameters can be verified on adsorption columns on the scale of a few milligrams (ZLC) to a 12-column system (RWA) with 1-2 kg of adsorbent. Through the validation of the model over 6 orders of magnitude in adsorbent amounts, the model can then be used to predict reliably the performance of adsorption systems on an industrial scale.

The design of a novel lab-scale rotary wheel adsorber has been presented for the first time. An industrial process of similar design would enable rapid thermal-swing adsorption alongside an efficiently integrated heat cycle. The rotary wheel adsorber in combination with novel materials having fast kinetics will enable fast adsorption cycle times and an overall reduction in the size of the capture plant. It is important that both the design of the adsorbents and the process are carried 
(a)

13X - Adsorbed concentration along the column

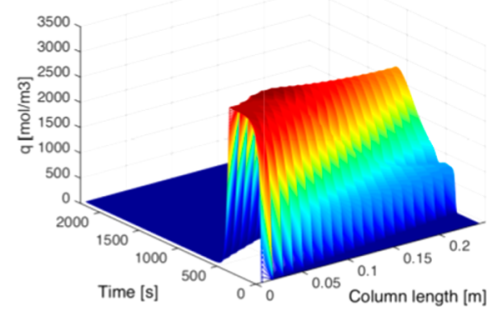

(b)

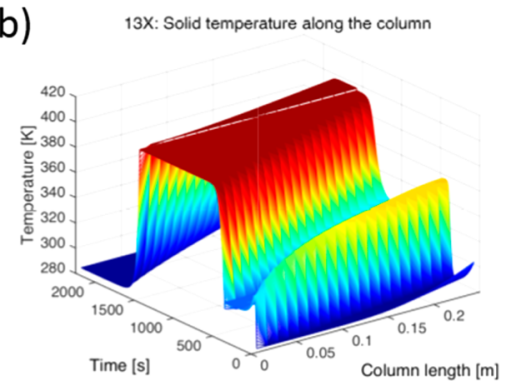

MCM-41-PEl: Adsorbed concentration along the column

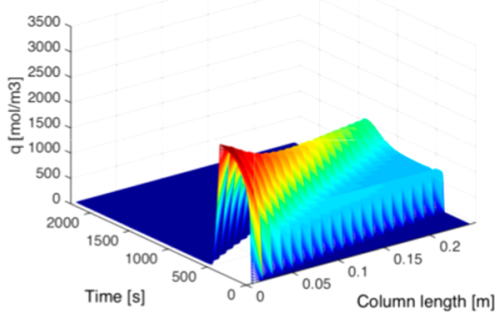

MCM-41-PEI: Solid temperature along the column

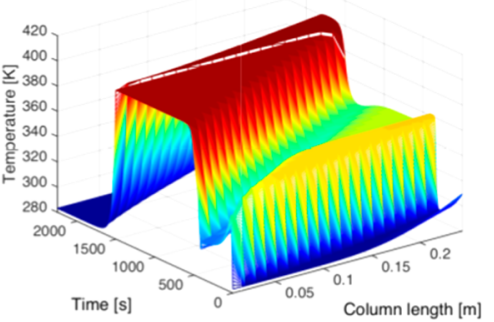

Figure 12. (a) $\mathrm{CO}_{2}$ breakthrough profiles along the column for $13 \mathrm{X}$ and MCM-41-PEI. (b) Temperature profiles of columns along the bed during adsorption, desorption and cooling.

Table 1. Summary of the Parameters Extracted for the Simple Process Configuration Using 13X and MCM-41-PEI as Adsorbents

\begin{tabular}{lll}
\multicolumn{1}{c}{ parameters } & \multicolumn{1}{c}{$13 \mathrm{X}$} & $\mathrm{MCM}-41-\mathrm{PEI}$ \\
cycle time $(\mathrm{s})$ & 2270 & 2270 \\
adsorption time $(\mathrm{s})$ & 409.96 & 409.96 \\
desorption time $(\mathrm{s})$ & 1090 & 1090 \\
cooling time $(\mathrm{s})$ & 769.98 & 769.98 \\
$F_{\text {feed }}\left(\frac{L / \text { min }}{\mathrm{kg}_{\text {adsorbent }}}\right)$ & 72.88 & 42.38 \\
$\mathrm{~F}_{\text {purge }}\left(\frac{L / \text { min }}{\mathrm{kg}_{\text {adsorbent }}}\right)$ & & \\
$\mathrm{F}_{\mathrm{N}_{2}}\left(\frac{L / \text { min }}{\mathrm{kg}_{\text {adsorbent }}}\right)$ & 51.69 & 51.69 \\
${\text { steam required }\left(\mathrm{kg} \text { of steam } / \mathrm{kg} \mathrm{CO} \mathrm{CO}_{2} \text { captured }\right)}_{\left.\mathrm{CO}_{2} \text { recovery } \%\right)}$ & 6.45 & 11.1 \\
$\mathrm{CO}_{2}$ purity $(\%)$ & 90 & 90 \\
\hline
\end{tabular}

out together to ensure that the process conditions are optimized to take full advantage of the adsorbents' properties and that the materials selected can be effective under process conditions.

\section{ASSOCIATED CONTENT}

\section{S Supporting Information}

The Supporting Information is available free of charge on the ACS Publications website at DOI: 10.1021/acs.iecr.5b05015.

Schematic of synthetic method of covalent functionalization of carbon surface, $\mathrm{CO}_{2}$ isotherms on $4 \mathrm{~mm} 13 \mathrm{X}$ pellets measure at 298,308 , and $323 \mathrm{~K}$, and $\mathrm{N}_{2}$ isotherms on $13 \mathrm{X}$ measured at 298,308 , and $323 \mathrm{~K}$ (PDF)

\section{AUTHOR INFORMATION}

\section{Corresponding Author}

*s.brandani@ed.ac.uk.

\section{Notes}

The authors declare no competing financial interest.

\section{ACKNOWLEDGMENTS}

This contribution was identified by Dr. Camille Petit (Imperial College London, U.K.) as the Best Presentation in the session "ENFL: Carbon Management: Recent Advances in Carbon Capture, Conversion, Utilization \& Storage" of the 2015 ACS Fall National Meeting in Boston, MA. Financial support from

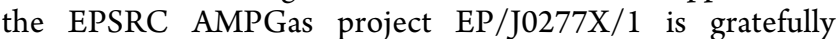
acknowledged, and to comply with RCUK requirements, the raw experimental data used in the manuscript can be found at http://dx.doi.org/10.7488/ds/1328.

\section{REFERENCES}

(1) Luo, X.; Wang, M.; Chen, J. Heat integration of natural gas combined cycle power plant integrated with post-combustion $\mathrm{CO}_{2}$ capture and compression. Fuel 2015, 151, 110-117.

(2) Abanades, J. C.; Arias, B.; Lyngfelt, A.; Mattisson, T.; Wiley, D. E.; Li, H.; Ho, M. T.; Mangano, E.; Brandani, S. Emerging $\mathrm{CO}_{2}$ capture systems. Int. J. Greenhouse Gas Control 2015, 40, 126-166.

(3) Sipöcz, N.; Tobiesen, F. A. Natural gas combined cycle power plants with $\mathrm{CO}_{2}$ capture - Opportunities to reduce cost. Int. J. Greenhouse Gas Control 2012, 7, 98-106.

(4) Lindqvist, K.; Jordal, K.; Haugen, G.; Hoff, K. A.; Anantharaman, $\mathrm{R}$. Integration aspects of reactive absorption for post-combustion $\mathrm{CO}_{2}$ capture from NGCC (natural gas combined cycle) power plants. Energy 2014, 78, 758-767.

(5) Boot-Handford, M.; Abanades, C.; Anthony, E.; Blunt, M. J.; Brandani, S.; Mac Dowell, N.; Fernández, J. R.; Ferrari, M.-C.; Gross, R.; Hallett, J. P.; Haszeldine, S.; Heptonstall, P.; Lyngfelt, A.; Makuch, Z.; Mangano, E.; Porter, R. T. J.; Pourkashanian, M.; Rochelle, G. T.; Shah, N.; Yao, J.; Fennell, P. Carbon Capture and Storage Update. Energy Environ. Sci. 2014, 7, 130-189.

(6) Samanta, A.; Zhao, A.; Shimizu, G. K. H.; Sarkar, P.; Gupta, R. Post-Combustion $\mathrm{CO}_{2}$ Capture Using Solid Sorbents: A Review. Ind. Eng. Chem. Res. 2012, 51, 1438-1463.

(7) Choi, S.; Drese, J. H.; Jones, C. W. Adsorbent Materials for Carbon Dioxide Capture from Large Anthropogenic Point Sources. ChemSusChem 2009, 2, 796-854.

(8) Ruthven, D. M.; Farooq, S.; Knaebel, K. S. Pressure Swing Adsorption; VCH Publishers: New York, 1994.

(9) Ruthven, D. M. Principles of Adsorption and Adsorption Processes; John Wiley \& Sons: New York, 1984. 
(10) Moon, S.-H.; Shim, J.-W. A novel process for $\mathrm{CO}_{2} / \mathrm{CH}_{4}$ gas separation on activated carbon fibers-electric swing adsorption. J. Colloid Interface Sci. 2006, 298, 523-528.

(11) Kodama, A.; Goto, M.; Hirose, T.; Kuma, T. Performance Evaluation for a Thermal Swing Honeycomb Rotor Adsorber Using a Humidity Chart. J. Chem. Eng. Jpn. 1995, 28, 19-24.

(12) Inventys VeloxoTherm Breakthrough Carbon Capture Technology. http://www.inventysinc.com/wp-content/uploads/2012/02/ Inventys-Brochure-Nov-2011-V8.pdf.

(13) Friedrich, D.; Ferrari, M. C.; Brandani, S. Efficient Simulation and Acceleration of Convergence for a Dual Piston Pressure Swing Adsorption System. Ind. Eng. Chem. Res. 2013, 52, 8897-8905.

(14) Hu, X.; Brandani, S.; Benin, A. I.; Willis, R. R. Development of a Semiautomated Zero Length Column Technique for Carbon Capture Applications: Rapid Capacity Ranking of Novel Adsorbents. Ind. Eng. Chem. Res. 2015, 54, 6772-6780.

(15) Mangano, E.; Brandani, S.; Ferrari, M. C.; Ahn, H.; Friedrich, D.; Lozinska, M. L.; Wright, P. A.; Kahr, J.; Morris, R.; Croad, M.; McKeown, N.; Shamsipour, H.; Budd, P. Efficient and Rapid Screening of Novel Adsorbents for Carbon Capture in the UK IGSCC Project. Energy Procedia 2013, 37, 40-47.

(16) Eic, M.; Ruthven, D. M. A new experimental technique for measurement of intracrystalline diffusivity. Zeolites 1988, 8, 40-45.

(17) Brandani, F.; Ruthven, D.; Coe, C. G. Measurement of Adsorption Equilibrium by the Zero Length Column (ZLC) Technique Part 1: Single-Component Systems. Ind. Eng. Chem. Res. 2003, 42, 1451-1461.

(18) Friedrich, D.; Mangano, E.; Brandani, S. Automatic estimation of kinetic and isotherm parameters from ZLC experiments. Chem. Eng. Sci. 2015, 126, 616-624.

(19) Brandani, F.; Ruthven, D. Measurement of Adsorption Equilibria by the Zero Length Column (ZLC) Technique Part 2: Binary Systems. Ind. Eng. Chem. Res. 2003, 42, 1462-1469.

(20) Guo, P.; Shin, J.; Greenaway, A. G.; Min, J. G.; Su, J.; Choi, H. J.; Liu, L.; Cox, P. A.; Hong, S. B.; Wright, P. A.; Zou, X. A zeolite family with expanding structural complexity and embedded isoreticular structures. Nature 2015, 524, 74-78.

(21) Lozinska, M. M.; Mangano, E.; Mowat, J. P. S.; Shepherd, A. M.; Howe, R. F.; Thompson, S. P.; Parker, J. E.; Brandani, S.; Wright, P. A. Understanding Carbon Dioxide Adsorption on Univalent Cation Forms of the Flexible Zeolite Rho at Conditions Relevant to Carbon Capture from Flue Gases. J. Am. Chem. Soc. 2012, 134, 17628-17642.

(22) Hu, X.; Mangano, E.; Friedrich, D.; Ahn, H.; Brandani, S. Diffusion mechanism of $\mathrm{CO}_{2}$ in $13 \mathrm{X}$ zeolite beads. Adsorption 2014, 20, 121-135.

(23) Hu, X.; Brandani, S.; Benin, A. I.; Willis, R. R. Development of a Semiautomated Zero Length Column Technique for Carbon Capture Applications: Study of Diffusion Behavior of $\mathrm{CO}_{2}$ in MOFs. Ind. Eng. Chem. Res. 2015, 54, 5777-5783.

(24) Brandani, S.; Ruthven, D. Analysis of ZLC desorption curves for gaseous systems. Adsorption 1996, 2, 133-143.

(25) Ruthven, D.; Brandani, F. ZLC response for systems with surface resistance control. Adsorption 2005, 11, 31-34.

(26) Kahr, J.; Morris, R. E.; Wright, P. A. Post-synthetic incorporation of nickel into $\mathrm{CPO}-27(\mathrm{Mg})$ to give materials with enhanced permanent porosity. CrystEngComm 2013, 15, 9779-9786.

(27) Lozinska, M. M.; Mowat, J. P. S.; Wright, P. A.; Thompson, S. P.; Jorda, J. L.; Palomino, M.; Valencia, S.; Rey, F. Cation Gating and Relocation during the Highly Selective "Trapdoor" Adsorption of $\mathrm{CO}_{2}$ on Univalent Cation Forms of Zeolite Rho. Chem. Mater. 2014, 26, 2052-2061.

(28) Greenaway, A. G.; Shin, J.; Cox, P. A.; Shiko, E.; Thompson, S. P.; Brandani, S.; Hong, S. B.; Wright, P. A. Structural changes of synthetic paulingite (Na,H-ECR-18) upon dehydration and $\mathrm{CO}_{2}$ adsorption. Z. Kristallogr. - Cryst. Mater. 2015, 230, 223.

(29) Greenaway, A.; Gonzalez-Santiago, B.; Donaldson, P. M.; Frogley, M. D.; Cinque, G.; Sotelo, J.; Moggach, S.; Shiko, E.; Brandani, S.; Howe, R. F.; Wright, P. A. In situ Synchrotron IR Microspectroscopy of $\mathrm{CO}_{2}$ Adsorption on Single Crystals of the
Functionalized MOF $\mathrm{Sc}_{2}\left(\mathrm{BDC}_{-} \mathrm{NH}_{2}\right)_{3}$. Angew. Chem., Int. Ed. 2014, 53, 13483-13487.

(30) Pillai, R. S.; Benoit, V.; Orsi, A.; Llewellyn, P. L.; Wright, P. A.; Maurin, G. Highly Selective $\mathrm{CO}_{2}$ Capture by Small Pore ScandiumBased Metal-Organic Frameworks. J. Phys. Chem. C 2015, 119, 23592-23598.

(31) Mowat, J. P. S.; Miller, S. R.; Griffin, J. M.; Seymour, V. R.; Ashbrook, S. E.; Thompson, S. P.; Fairen-Jimenez, D.; Banu, A.-M.; Düren, T.; Wright, P. A. Structural Chemistry, Monoclinic-toOrthorhombic Phase Transition, and $\mathrm{CO}_{2}$ Adsorption Behavior of the Small Pore Scandium Terephthalate, $\mathrm{Sc}_{2}\left(\mathrm{O}_{2} \mathrm{CC}_{6} \mathrm{H}_{4} \mathrm{CO}_{2}\right)_{3}$, and Its Nitro- and Amino-Functionalized Derivatives. Inorg. Chem. 2011, 50, 10844-10858.

(32) Gibson, J. A. A.; Gromov, A. V.; Brandani, S.; Campbell, E. E. B. The effect of pore structure on the $\mathrm{CO}_{2}$ adsorption efficiency of polyamine impregnated porous carbons. Microporous Mesoporous Mater. 2015, 208, 129-139.

(33) Zhao, J.; Simeon, F.; Wang, Y.; Luo, G.; Hatton, T. A. Polyethylenimine-impregnated siliceous mesocellular foam particles as high capacity $\mathrm{CO}_{2}$ adsorbents. RSC $A d v$. 2012, 2 (16), 6509-6519.

(34) Dang, W.; Friedrich, D.; Brandani, S. Characterisation of an Automated Dual Piston Pressure Swing Adsorption (DP-PSA) System. Energy Procedia 2013, 37, 57-64.

(35) Ruthven, D. M.; Thaeron, C. Performance of a parallel passage adsorbent contactor. Gas Sep. Purif. 1996, 10, 63-73.

(36) Tsonev, D.; Videv, S.; Haas, H. SPIE OPTO, 2013; International Society for Optics and Photonics. Proc. SPIE 2014, 900702.

(37) Afgani, M. Z.; Haas, H.; Elgala, H.; Knipp, D. Visible light communication using OFDM. In 2nd International Conference on Testbeds and Research Infrastructures for the Development of Networks and Communities, 2006: TRIDENTCOM 2006; IEEE: New York, 2006; pp 129-134.10.1109/TRIDNT.2006.1649137

(38) Belmabkhout, Y.; Serna-Guerrero, R.; Sayari, A. Adsorption of $\mathrm{CO}_{2}$-Containing Gas Mixtures over Amine-Bearing Pore-Expanded MCM-41 Silica: Application for Gas Purification. Ind. Eng. Chem. Res. 2010, 49, 359-365. 